\title{
Bragg spectroscopy from CORONAS-F
}

\author{
J. Sylwester \\ Space Research Centre, Polish Academy of Sciences, Wroclaw ul. Kopernika 11, Poland \\ email: js@cbk.pan.wroc.pl
}

\begin{abstract}
There are dozen instruments successfully operating aboard the solar satellite CORONAS$F$, which was launched in the summer of 2001. Among them are two (Polish-led) Bragg crystal spectrometers - RESIK and Diogeness - recording solar flare and active region spectra. A short description of the CORONAS-F satellite operation is presented together with that of the two Polish spectrometers, stressing their unique characteristics. The average spectra have been derived and shown here, covering the wavelength range between $3 \AA$ and $7 \AA$. Future steps in the analysis of the large database collected are outlined.
\end{abstract}

\section{Introduction}

In the past 40 years it has become clear that progress in understanding the mechanism of coronal energy release can be obtained using data fromspectrometers operating in the soft X-ray region. These observations provide valuable information about the temperature, ionization state, nonthermal and directed plasma motions. Among the spectrometers used in this spectral range the scanning, flat crystal spectrometers on the early NASA Orbiting Solar Observatories, the U.S. Navy P78-1 satellite, NASA's Solar Maximum Mission (SMM: the X-ray Polychromator/Flat Crystal Spectrometer), and the Japanese Hinotori spacecraft contributed the most to our understanding of the spectral variability. However, because of the length of time needed to scan particular portions of the X-ray spectrum, critical phases of flares were often missed with these spectrometers. Curved crystal spectrometers like the SMM Bent Crystal Spectrometer and the Bragg Crystal Spectrometer (BCS) on the Japanese Yohkoh spacecraft have an important advantage in this respect since they are designed to take complete spectra, generally over limited wavelength bands, over time intervals of only a few seconds. With strong emission lines like the resonance lines of highly ionized $\mathrm{Ca}$ and $\mathrm{Fe}$, formed at high temperatures, spectral changes and thus information about physical conditions during the rapid development of particular flares can be followed (Antonucci, 1989).

The analysis of results obtained from these earlier spectroscopic solar missions led us to the idea of verifying, this time in absolute terms, the determinations of Doppler shifts observed early in flares. We tested in the sounding rocket flight (Vertical-11, 1983) the concept of a new type of scanning Bragg X-ray spectrometer - so called Dopplerometer and decided, following its success to devise a satellite version of this instrument called Diogeness (Sylwester \& Farnik, 1990). Diogenes flew on CORONAS-I, however, the mission ended before really big flares took place and no useful spectra were recorded. On CORONAS-F we were more successful and acquired many spectra as reported below.

In preparing our programme of experimental spectroscopic studies of the corona, we have noticed that large "gaps" are still present in detailed observations of solar spectra in the soft X-range, below $\sim 7 \AA$. Therefore we decided to devise a new spectrometer intended to cover this spectral region with observations in a systematic way. In collaboration with leading groups from Naval ResearchLaboratory, USA (NRL), Mullard Space 


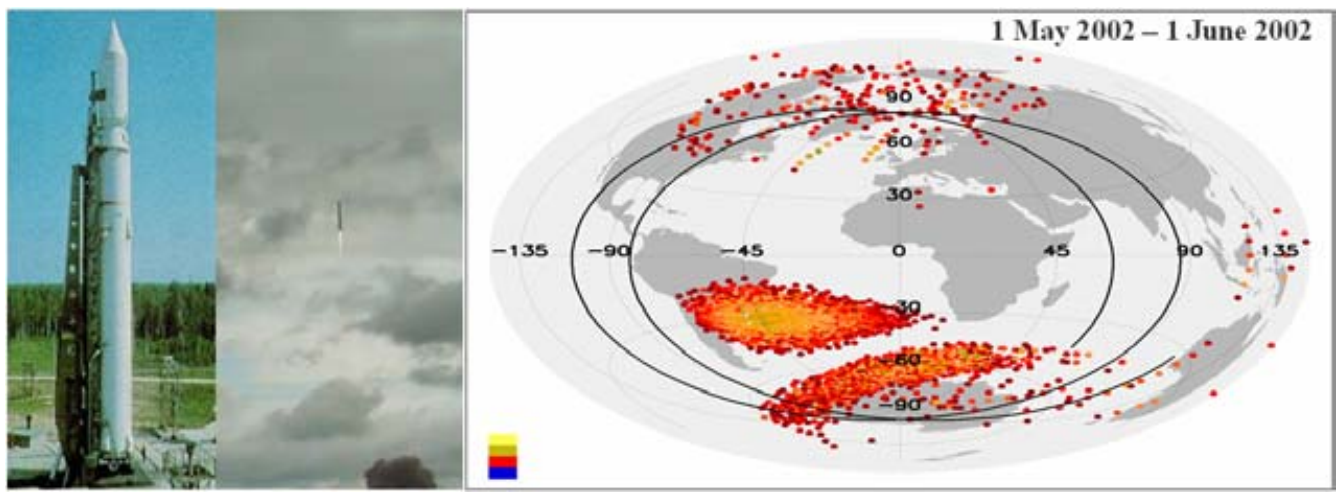

Figure 1. A composite showing the Cyclone 3 rocket booster which lifted the CORONAS-F satellite on 31 June 2001. In the right panel, the satellite orbit is plotted over the map showing locations of increased orbital background. The map is constructed based on the data from the PIN particle sensors placed within RESIK spectrometer.

Science Laboratory (MSSL) and Rutherford-Appleton Laboratory (RAL, both from UK) we constructed REntgenovsky Spektrometr s Izognutymi Kristalami (RESIK), the bent crystal spectrometer, described in details by Sylwester et al. (2004). Both Diogeness and RESIK have been launched aboard the Russian CORONAS-F solar spacecraft on 31 July 2001 (Sylwester \& Kordylewski, 2002).

In this paper we show the average spectra observed from these spectrometers. The spectra shown include yet unobserved regions.

\section{CORONAS-F satellite}

The CORONAS-F spacecraft is the second of two Russian space observatories devoted to solar and magnetospheric physics, designed to be operational during the 23rd solar cycle. Its primary objectives are the study of solar global oscillations, variations in the solar ultraviolet radiation, and flare and active region X-ray, gamma-ray, and particle emission. Except Polish spectrometers which results are presented here, there are dozen of Russian instruments, some of them presented in more details also in the other contributions of the present Volume.

The CORONAS-F spacecraft is in a near-circular orbit with an altitude ranging from $501 \mathrm{~km}$ and $549 \mathrm{~km}$, with an orbital plane inclined at $82^{\circ} .5$ to the equator, and orbital period of 95 minutes. The near-polar orbit allows periods of uninterrupted observations of the Sun for up to about 20 days: the longest satellite night lasts 35 minutes. Passages through the Earth's radiation belts require most X-ray instruments to be turned off in order to secure their health; these include passages through the South Atlantic Anomaly (SAA) as well as the auroral oval (AO) regions near the magnetic poles. In figure 1, the satellite orbit is plotted over the Earth map, with the regions of enhanced particle background indicated.

\section{Diogeness spectra}

Diogeness is the scanning flat crystal spectrometer. The scanning range covers $\sim 140$ arcmin. Details concerning the crystal selection and wavelength ranges are given in table 1. The spectrometer is composed of four crystals. Two of the crystals used are identical quartz mounted in the so-called Dopplerometer configuration (figure 5) back to back. 


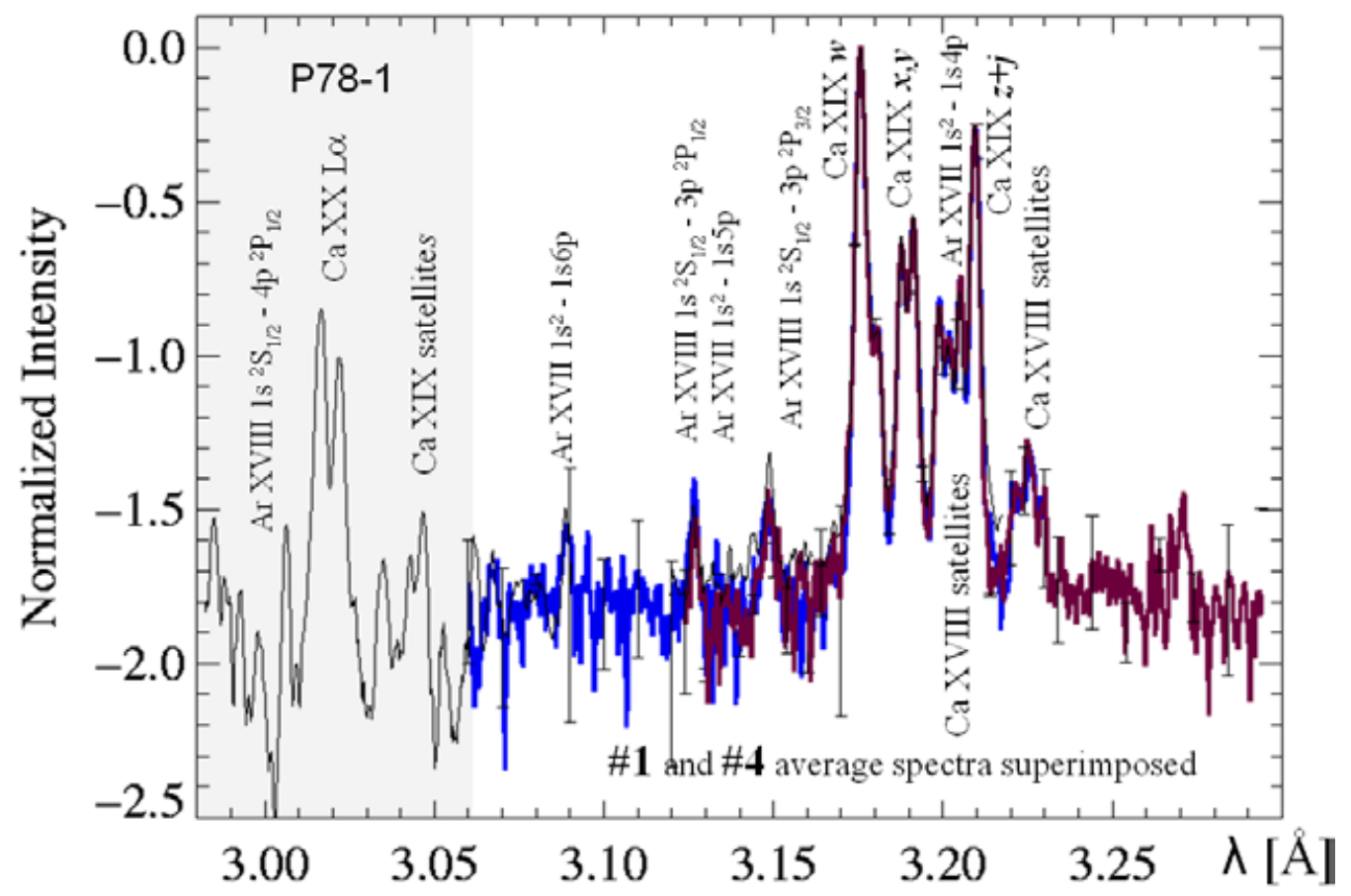

Figure 2. Diogeness average normalized (to maximum) spectrum in the range covered by channels Nos. 1 and 4. Logarithmic scaling is used on the vertical axis in order to bring-up weaker spectral structures. Earlier results (gray area) obtained from the P78-1 experiment (Doschek, Feldman \& Seely, 1985) are merged using the overlapping part of the continuum as a common reference. This merging allows to see in one piece the entire spectral range covering the $\mathrm{Ca} \mathrm{XX}$ Ly $\alpha$ region as well as the Ca XIX He-like triplet with their numerous satellite lines.

$\begin{array}{lcccc} & \text { Channel 1 } & \text { Channel 2 } & \text { Channel 3 } & \text { Channel 4 } \\ \text { Crystal } & \text { Quartz } & \text { ADP } & \text { Beryl } & \text { Quartz } \\ \text { plane } & 10 \overline{1} 1 & 111 & 10 \overline{10} & 10 \overline{1} 1 \\ \begin{array}{l}\text { 2d spacing } \\ \text { Wavelength range }[\AA]\end{array} & 6.69 & 10.57 & 15.96 & 6.69 \\ \begin{array}{l}\text { Principal } \\ \text { lines in range }\end{array} & 3.14-3.39 & 4.98-5.37 & 6.11-6.73 & 2.96-3.21 \\ & \text { Ca XIX } & \text { S XVI } & \text { Si XIII } & \text { Ca XIX }\end{array}$

Table 1. Characteristics of Diogeness spectrometer

Therefore the wavelength scanning is taking place in the opposite sense. Such arrangement of the crystals allows for precise relative and absolute measurements of Doppler shifts of selected X-ray lines. For plasma at rest, the maxima of lines scanned are being measured approximately at the same instant. If any radial motions are present, the lines are observed off-set in time. The off-set is directly related to the plasma radial velocity, independent of the position of the flare on the solar disk. The observed widths of spectral lines (figures $2,3 \& 4$ ) reflect mostly the intrinsic line widths (thermal and non-thermal broadening) since the instrumental rocking curves widths are much smaller. Since the four crystal-detector sections are equipped with a small proportional detector windows $\left(0.3 \mathrm{~cm}^{2}\right.$ area) covered with Be $130 \mu \mathrm{m}$ filter, only larger flares (above C5 GOES class) provide spectral signal above background. Even in the strongest X-class flares, the detectors did not get saturated. Hundreds of spectra from particularly well observed X5.3 flare 


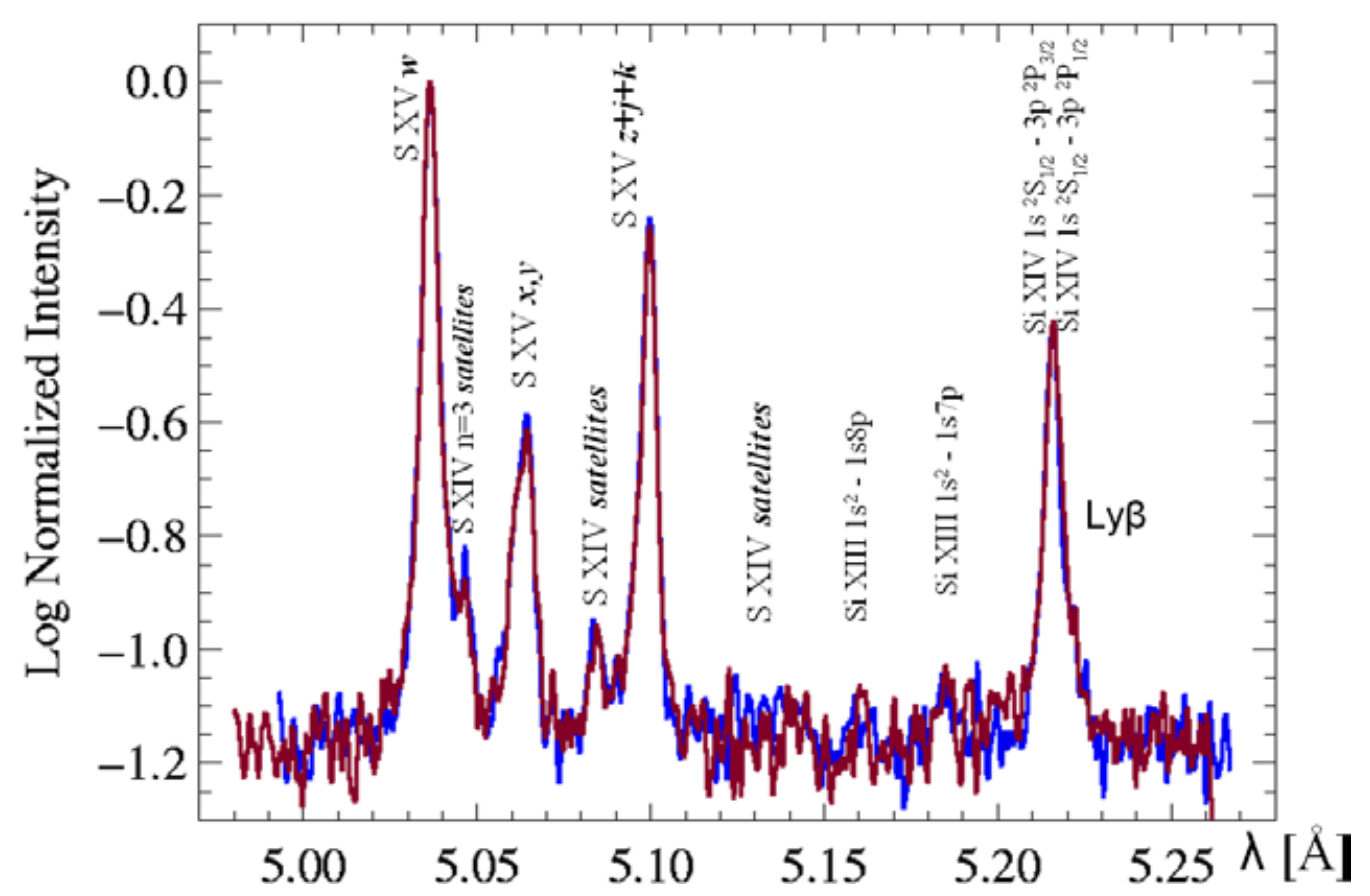

Figure 3. Diogeness average normalized (to maximum) spectra in the range covered by channel No. 2. Logarithmic scaling is used on the vertical axis in order to bring-up weaker spectral structures. The spectrum in the vicinity of S Xv He-like ion triplet is seen in detail as well as the Si XIV Ly $\alpha$ region with their numerous satellite lines. The two overlapping lines represent spectra as averaged from the left and the right scans respectively.

on 25 August 2001 seen by Diogeness include strong emission lines of highly ionized Si, $\mathrm{S}$, and Ca atoms as well as many satellite and subordinate lines. In figures 2, 3 and 4 we present average spectra recorded for this X5.3 flare. The time averaging extends over the rise, maximum and decay phases with each scanned spectrum taken with equal weight. The averaging allows to see combined spectral features of the hotter (rise and maximum) and cooler plasmas (during flare decay) together. Averaged spectra from so-called left and right scans (in which the scanning took place in the opposite wavelength sense) we plot in logarithmic scale on-top each other. The differences between the left and right averaged spectra are usually so small that the separation of lines is mostly undistinguishable. We are confident of the physical significance of even the weakest features seen, provided the two profiles match. In figure 2, we supplement the Diogeness spectra with those measured earlier by the NRL group (Doschek, Feldman, \& Seely, 1985).

Besides the strongest He-like triplet lines, Diogeness measures also the other lines important for plasma diagnostics. One of the most interesting is the Ly $\beta$ line of Si XIV ion at $5.216 \AA$ (figure 3 ). As this line is mostly formed in the hottest regions of the flare, its intensity is observed to fall rapidly during the decay (Siarkowski et al., 2002). Several features seen on the presented spectra are observed for the first time. Their identification is still tentative.

As concerns the results coming from the Dopplerometer section, with all the satellite drift motions and the other instrumental effects found unimportant (Plocieniak et al., 2002), the relative observed shifts of the line positions in respect to the bi-sector plane have been interpreted as a consequence of the Doppler effect. The results are plotted in figure 5 for the X5.3 flare on 25 August 2001. The velocity determinations have been performed independently from the shifts observed for the resonance lines and blends of 


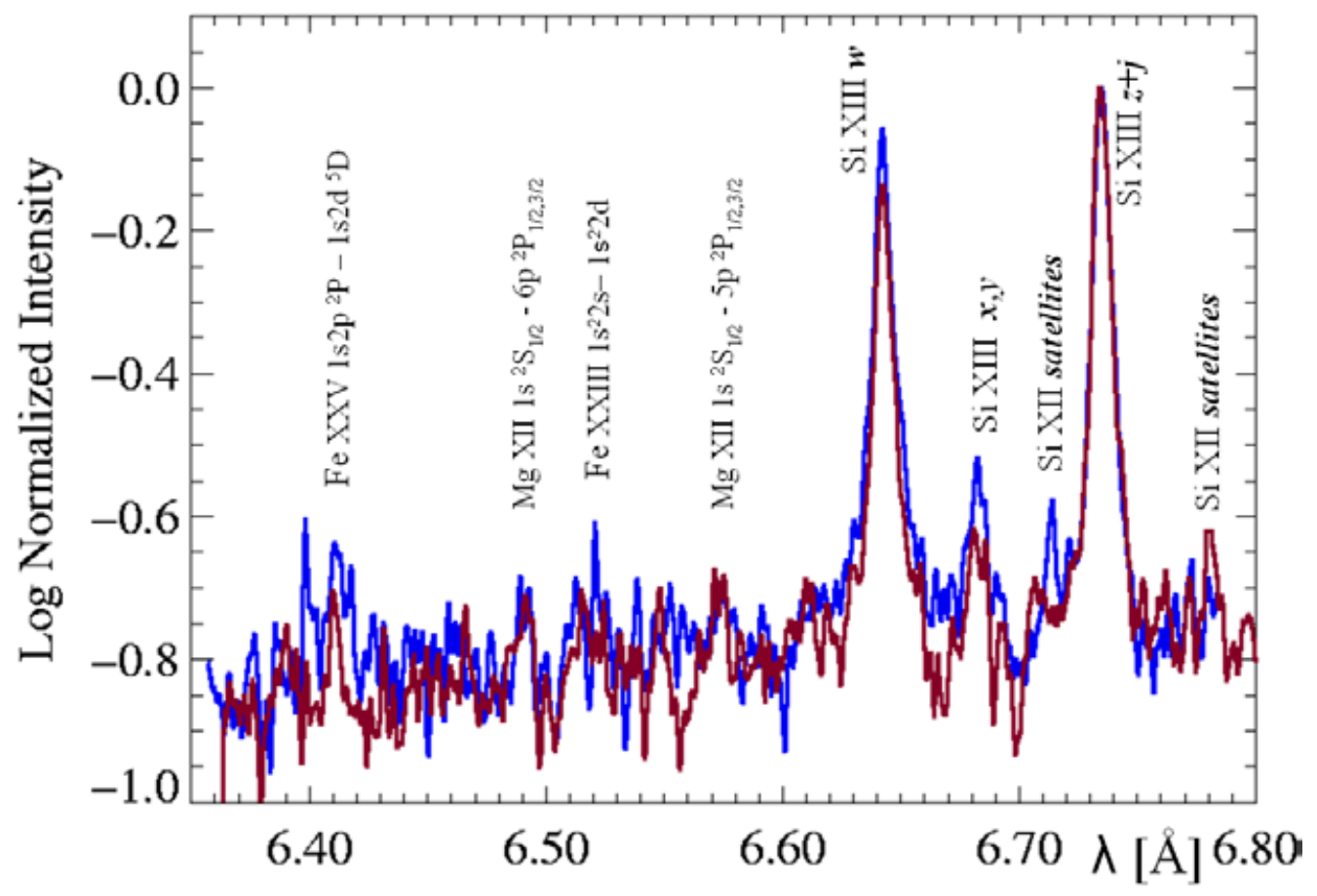

Figure 4. Diogeness average normalized (to maximum) spectra in the range covered by channel No. 3. Logarithmic scaling is used on the vertical axis in order to bring-up weaker spectral structures. The spectrum in the vicinity of Si XIII He-like ion triplet is seen in detail. The two overlapping lines represent spectra as averaged from left and right scans respectively.

forbidden with satellite lines of Ca XIX (lines $w$ and $z+j$ respectively in figure 2). It is seen that these independent determinations compare well to the level of the uncertainty of velocity determinations (few $\mathrm{km} / \mathrm{s}$ ). This agreement provides strong argument in favour that for the observed flare, the entire X-ray spectra were Doppler shifted by $\sim 100 \mathrm{~km} / \mathrm{s}$ for Ca XIX ion abundant at $T>15$ MK. Similar pattern of shifts have been observed for cooler plasma contributing to S XV and Si XIII ions line emission (Plocieniak et al., 2002).

\section{RESIK spectra}

RESIK is the bent crystal spectrometer recording all wavelengths at the same instant in four spectral channels. Details concerning the crystal used and the spectral coverage are given in table 2 . The observed widths of spectral lines (figures $6 \& 7$ ) reflect the instrumental line widths as the spectral bin has to be wide enough in order to cover the large spectral range selected. Full description of RESIK instrument is given in Sylwester et al., (2004). It is possible to observe with RESIK in higher order of reflections. In figure 6 , we show examples of the spectra collected in individual contiguous time intervals in the four spectral channels (first and third order reflections) before (as recorded) and after the reduction to the absolute units. RESIK primary wavelength coverage, $3.3 \AA-6.1 \AA$, has been very little explored by previous solar spacecraft. Spectra from solar flares seen by RESIK include strong emission lines of highly ionized Si, S, and Ar atoms, together with lines from the low-abundance, odd- $Z$ elements $\mathrm{K}$ and $\mathrm{Cl}$. Some of these lines are also seen in the spectra of brighter, non-flaring active regions and "quiet Sun". Several spectral features have been observed for the first time in solar X-ray spectra. In figure 7 

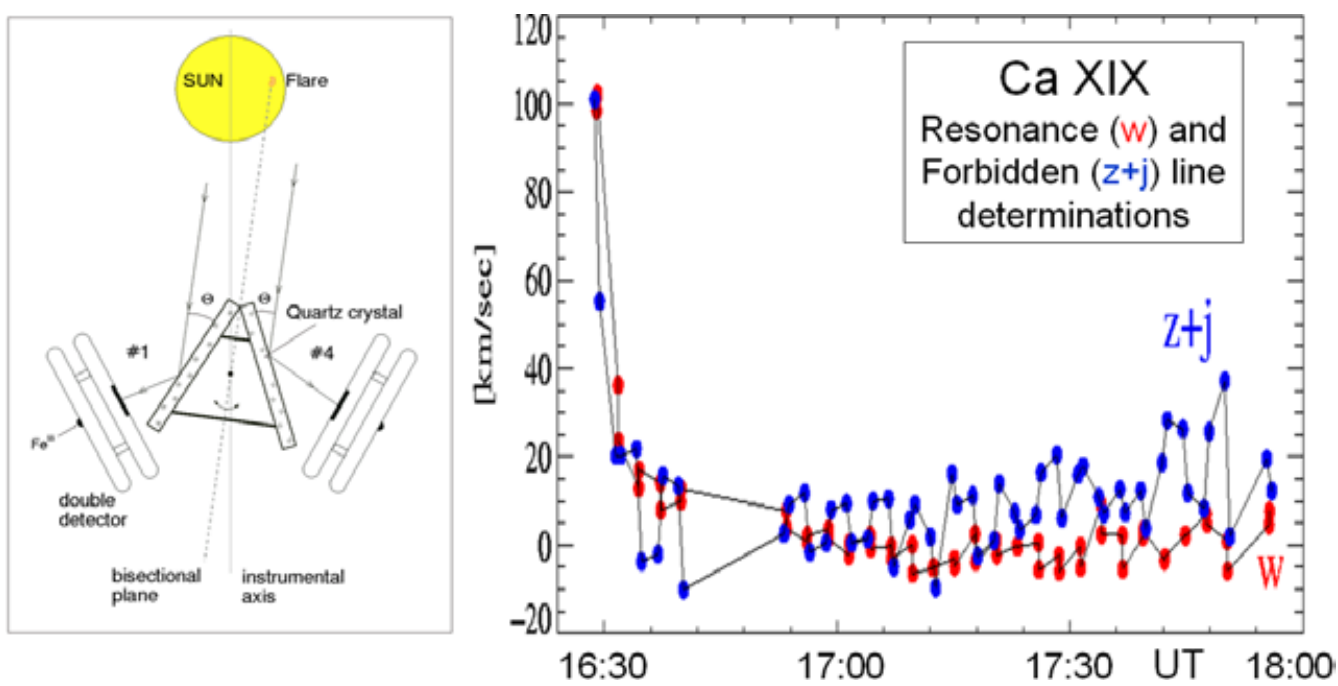

Figure 5. Left: The scheme of Dopplerometer configuration adopted in the experiment. Bi-sector plane of the crystal pair \#1 and \#4 (dotted line) points momentarily towards the source (flare). Crystals are fixed mechanically at the exact angle of $2 \Theta$. By rotating fixed crystal section back and forward, the Bragg-reflected spectra in the vicinity of strong lines are measured by a pair of double proportional counters. In the ideal case of perfect co-alignment and for the source plasma being at rest, the line centres are recorded in channels \#1 and \#4 at the very same time during the scans performed in the opposite wavelength sense. Any radial motions of the hot flaring plasma will displace (in time) the relative position of lines. The displacement is proportional to the line Doppler shift and is independent of the position of the source (flare) on the disc and/or the relative pointing, provided they are stable. Right: Velocities determined from X-ray line shifts observed by Diogeness. Determinations for Ca " $w$ " and Ca " $z+j$ "line are superimposed. A high blue-shift is observed early in flare. The shifts of entire spectra are of similar value in all lines measured. As the Ca " $w$ " line is not badly blended (as is the case of the forbidden line) the uncertainties of velocity determinations are few $\mathrm{km} / \mathrm{s}$ only for this line.

\begin{tabular}{|c|c|c|c|c|}
\hline Cryst & $\underset{\mathrm{Si}}{\text { Channel }} 1$ & $\underset{\mathrm{Si}}{\text { Channel }} 2$ & $\begin{array}{l}\text { Chan } \\
\text { Qua }\end{array}$ & Chat \\
\hline plane & 111 & 111 & $10 \overline{1} 0$ & 10 \\
\hline $\begin{array}{l}2 d \text { spacing } \\
\text { bend radius }\end{array}\left[\begin{array}{c}{[\AA]} \\
\mathrm{cm}]\end{array}\right.$ & 6.27 & 6.2 & & \\
\hline Wavelength range $^{a}[\AA]$ & $3.40-3.80$ & $3.83-4.27$ & $4.35-4.86$ & $5.00-6.05$ \\
\hline & 8 & 9 & 12 & 17 \\
\hline Dispersion $\quad[\mathrm{m} \AA / \mathrm{bin}]$ & 2.49 & 2.28 & 2.85 & 4.99 \\
\hline lines $i$ & $\begin{array}{l}\text { Ar XVIII, } \\
\text { K XVIII }\end{array}$ & $\underset{\text { S XV }}{\operatorname{Ar} \text { XVII, }}$ & S XVI & $\begin{array}{l}\text { KV, Si XI } \\
\text { Si XIII }\end{array}$ \\
\hline
\end{tabular}

${ }^{a}$ Nominal wavelength ranges are those recorded always

Table 2. Characteristics of RESIK spectrometer

we present a composite of the observed spectral variability in four RESIK channels. The reduced spectra shown are averages of many individual (from a sample of $~ 1200$ ) for five temperature categories. Before averaging, the spectra have been normalized to unit emission measure $\left(10^{44} \mathrm{~cm}^{-3}\right)$. The temperature has been assigned to each individual spectrum based on the interpretation of the signal ratio measured in channels No. 1 and No. 4. What appears unexpected is presence of few broad-band spectral features (bumps), systematically changing their appearance with temperature. Importance of bumps at 

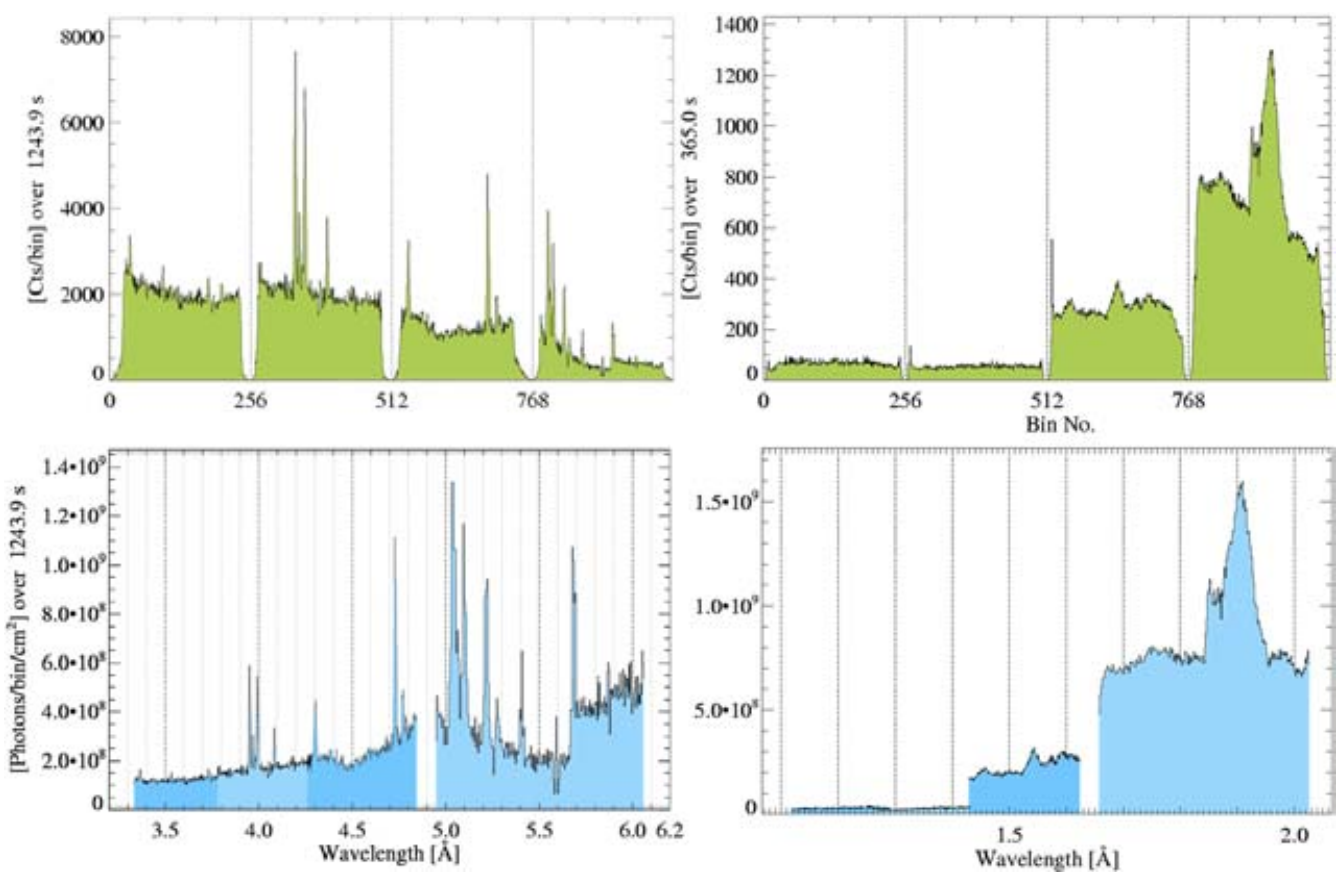

Figure 6. Left: Example composite RESIK spectra as measured (upper panel) and after reduction (lower panel). During the reduction process, orbital background and fluorescence contamination have been removed. The spectra shown were collected during the 20 -minute rise phase of the M1.9 East-limb flare on 2003 January 21. Right: Spectra measured in the mode where detectors were sensitive to photons reflected from the crystals in second and third order. The spectra shown were collected over $365 \mathrm{~s}$ during the maximum phase of the M2.1 flare on April 26, 2003.

$\lambda \lambda$ 3.60-3.85 $\AA$ 4.05-4.25 $\AA$ and 4.5-4.9 $\AA$ decreases with temperature, but the role of the bump at 5.6-6.0 $\AA$ grows with increasing $\mathrm{T}$. We are yet unsure of the processes contributing to presence of the observed bumps.

\section{Conclusions}

We present spectra recorded using two Bragg crystal spectrometers Diogeness and RESIK placed aboard the CORONAS-F solar orbiting observatory. Diogeness have observed hundreds of spectra in vicinity of triplet lines of He-like Ca, S and Si ions. The quality of spectra measured is excellent for few big flares observed, allowing for prompt identification of a number of spectral features rarely seen before. The sensitivity of the spectrometer is however not sufficient to record spectra from flares below c5 GOES class. For one flare analysed in details, systematic plasma upflows have been detected during the rise phase, which caused entire spectra including triplet and satellite lines formed in Ca XIX ion to be Doppler-shifted.

The spectra recorded cover yet unexplored spectral ranges with unprecedented resolution and constitute the reference spectral atlas in the range below $6.8 \AA$. Many spectra features including broad "bumps" has not been observed before. The variability of spectra with changing temperature is discussed which will lead to the identification of the spectral features seen. This will be the subject of forthcoming papers. All the figures presented here in black and white are available in colours at the address: http://www.cbk.pan.wroc.pl/publications/2004/IAU223_Bragg.html. 

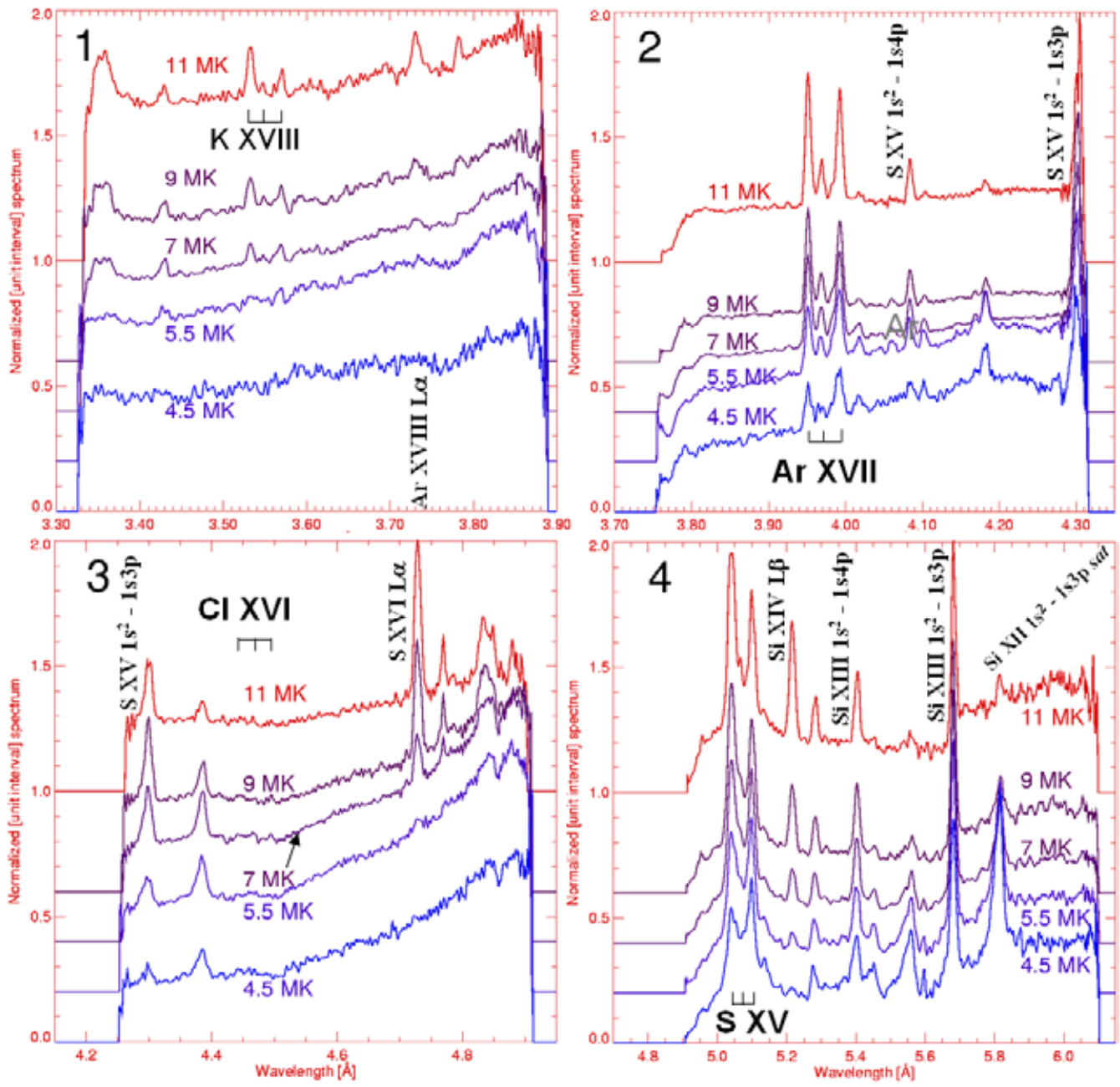

Figure 7. Composite showing temperature dependence of average spectra observed by RESIK in five temperature categories in all four first order channels. Spectra are normalised to their respective maxima and slighly vertically shifted (see the side levels) in order to increase visibility. Main lines are identified and positions of He-like triplets indicated.

\section{Acknowledgements}

I would like to thank Barbara Sylwester for useful discussions and help in preparing this review. We acknowledge financial support for RESIK from the Polish grants 2.P03D.002.22 and PBZ-KBN-054/P03/2001, and the Exchange Visitors Programme of The Royal Society-Polish Academy of Sciences.

\section{References}

Antonucci, E. 1989 Solar Phys. 121, 31-60.

Doschek, G.A., Feldman, U. \& Seely, J.F. 1985 Non. Not. Royal Astr. Soc. 217, 317-326.

Plocieniak, S., Sylwester, J., Kordylewski, Z. \& Sylwester, B. 2002 ESA SP 506, 963-966.

Siarkowski, M., Sylwester, J., Plocieniak, S., \& Kordylewski, Z. 2002 ESA SP 506, 753-756.

Sylwester, J. \& Fárnik 1990 Bull. Astron. Inst. of Czech. 41, 149-157

Sylwester, J.\& Kordylewski, Z. 2002 Adv. Space Res. 30, 105-110.

Sylwester, J.\& 21 co-authors 2004 Solar Phys. submitted 